\title{
GLOBAL STRUCTURE OF AN AXIALLY SYMMETRIC PULSAR MAGNETOSPHERE
}

\author{
Yu. A. RYLOV \\ Institute for Problems in Mechanics, Academy of Sciences
}

\section{Introduction}

A self-consistent model of the pulsar magnetosphere is presented. The principal point of the model is its use of the massless approximation. In this approximation electrons and positrons are considered as massless particles, and the pulsar magnetosphere consists of regions of two types: (1) accelerating regions (A-regions) filled with particles moving with the speed of light [dynamical phase (DP)], (2) capture regions (C-regions) which, besides the DP, contain static phase (SP), i.e. captured particles resting at some coordinate system.

The pulsar magnetosphere structure is determined by the shape of the C-regions (and Aregions). In the model presented their shape is calculated. In this respect the model presented differs from others (the models of the first generation), where the shape of the C-region is set. For instance, in the Goldreich and Julian model (1969) the capture region is supposed to occupy the whole space, which contradicts the dynamical equations and leads to singularities on the light cylinder.

From the mathematical standpoint the calculation of the capture region shape is possible due to the derivation of certain conditions on the boundary between the $\mathrm{C}$ and $\mathrm{A}$-regions. These conditions are a variety of surface dynamical equations (Rylov 1989).

The pulsar magnetosphere can be considered as a giant vacuum-tube device generating radio emission. The construction of this device and its operating regime are calculated in the model presented. This explains the manner of the radio-emission generation, the high brightness temperature of the pulsar radio emission, its sharp directivity, some general features of the pulsar radio spectrum and the flux of hard electrons and positrons from the pulsar magnetosphere.

The model is self-consistent. It has no singularities on the light cylinder and no other inconsistencies.

\section{The pulsar as a vacuum-tube device}

A pulsar is a rapidly rotating neutron star with a strong magnetic field $\boldsymbol{H}$. For simplicity the magnetic field is supposed to be dipolar, the magnetic dipole $\boldsymbol{\mu}$ being aligned with the angular velocity $\boldsymbol{\Omega}_{0}$ of the $\operatorname{star}\left(\boldsymbol{\Omega}_{0} \| \boldsymbol{\mu},\left(\boldsymbol{\Omega}_{0}, \boldsymbol{\mu}\right)>0\right)$. The pulsar magnetosphere considered as a vacuum-tube device has two controlling parameters: the total electric charge $Q$ and the radius $R$ of the maximal conducting corotating sphere around the star. In the simplest case $R$ coincides with the stellar radius $R^{*}$.

The construction and the operation of the vacuum-tube device can be understood most simply if electrons and positrons are considered as massless particles. In the accelerating region, where $(E, H) \neq 0$ or $E^{2} \geq H^{2}$, the massless charged particle moves with the speed of light, forming a dynamical phase (DP) (Rylov 1988). In the capture region, where $(\boldsymbol{E}, \boldsymbol{H})=0$ and $E^{2}<H^{2}$, it can move with the speed $|v|<c$, forming a static phase (SP) (Rylov 1988).

The structure of the pulsar magnetosphere is determined by the size and the shape of the capture regions. The pulsar magnetosphere is very sensitive to the total charge $Q$ of the system. For a sufficiently large $Q>0$ the pulsar magnetosphere is static (figure 1). This result was obtained in papers (Rylov 1976, Krausse-Pohlstorff and Michel 1985a) by means of numerical simulation.

If the total charge $Q$ decreases, the electron dome size increases. It reaches the light cylinder, and the pulsar magnetosphere stops being static. The pulsar magnetosphere structure for small $Q$ or $Q=0$ is presented in figure 2 .

Parameters of the main elements of the stationary magnetosphere were calculated in papers by Rylov (1977, 1979, 1985, 1989). The total charge $Q$ is determined from proper balance equations. With a sufficiently large charge $Q$ the magnetosphere is static. With a rather small or negative charge the electrons escape. The total charge regulates the flow of returning or escaping electrons. Inside the outflow channel the electron DP moves through the electron SP. The arising two-beam regime leads to bunching. Numerical simulation of this bunching 


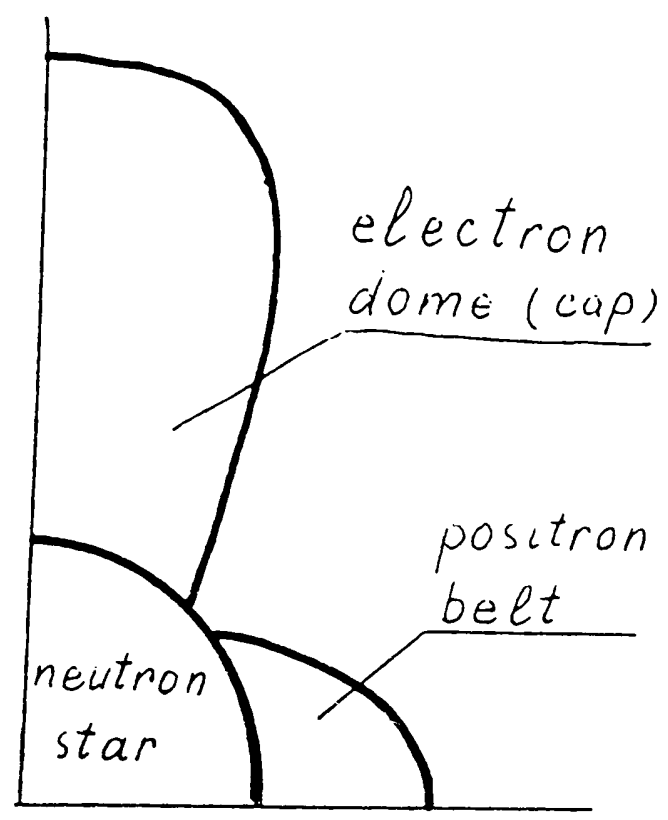

Figure 1 Static magnetosphere.

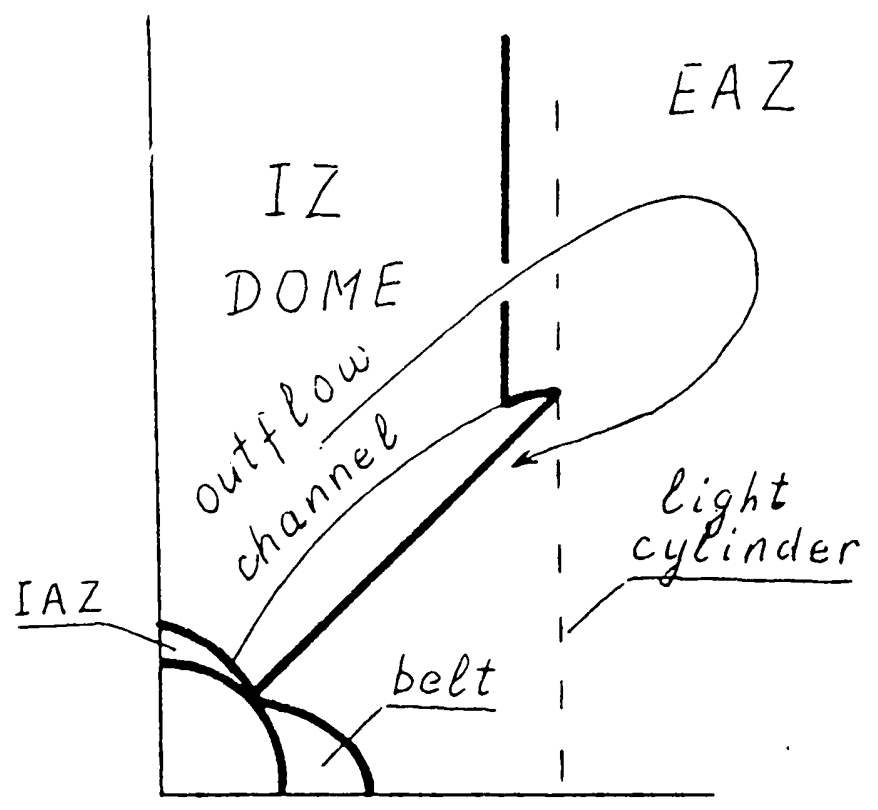

Figure 2 Magnetosphere for small $Q$.

shows that it is very strong (Rylov 1987).

Fluctuations of the electron density inside the outflow channel can be treated as a gas of electron bunches rather than small vibrations of a steadystate flow. The gas of electron bunches has a very high temperature due to the large mass of the bunches. It is a source of radio emission of high brightness temperature and sharp directivity. It should be stressed that the above mentioned selfconsistent magnetosphere is well founded mathematically. It is not only an assumption.

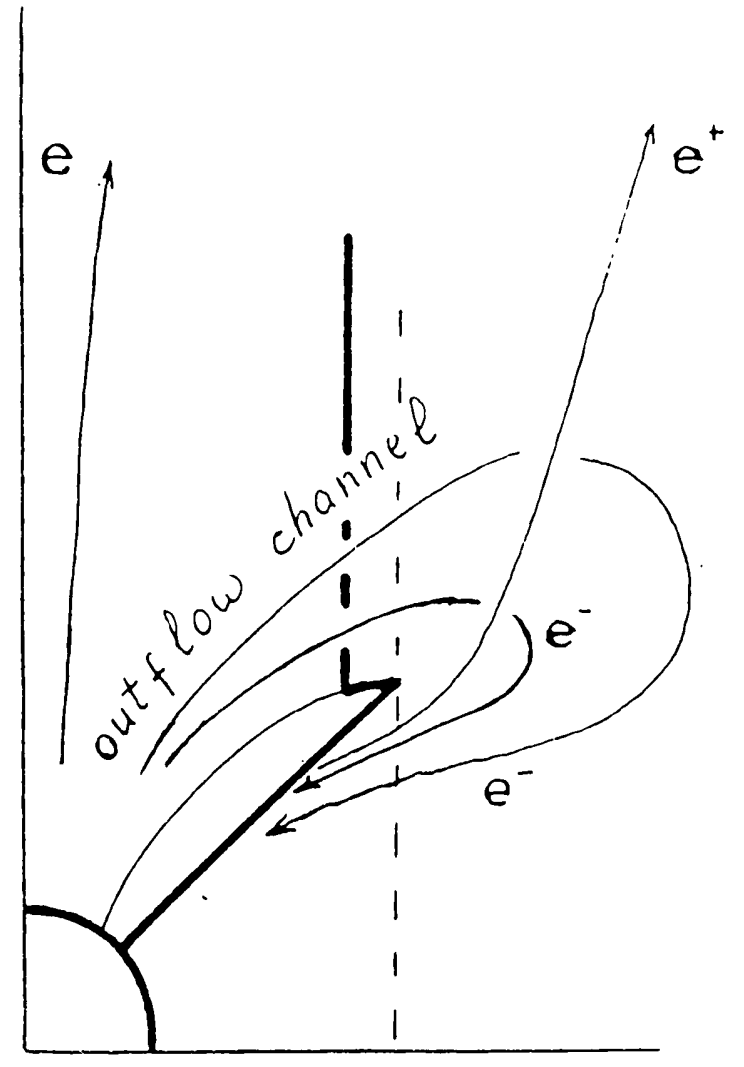

Figure 3

\section{Elements of the vacuum-tube device "pulsar"}

The pulsar magnetosphere consists of the following essential elements:

1. an equatorial positron belt,

2. an electron dome (cap),

3. the outflow channel, where one can distinguish parts:

(a) internal accelerating zone (IAZ) near the stellar surface,

(b) inertial zone (IZ), where electrons are not accelerated,

(c) external accelerating zone (EAZ), where electrons are accelerated towards the star up to very large Lorentz factors $10^{7}$ to $10^{9}$.

All elements were calculated in papers by Rylov $(1977,1979,1985)$ except the boundary between the $I Z$ and EAZ which was only estimated in Rylov (1989).

1. The positron belt is a capture region which consists of corotating and superrotating parts. The shape and the angular frequency of the 
superrotating part depend on the occupation number $k_{\text {oml }}$ of electrons on the magnetic line (Rylov 1985).

2. The shape of the lower part $(r<10 R)$ of the dome depends on the charge and the shape of the belt. The upper part of the dome is universal $(r \gg R)$. It does not depend on the shape or the charge of the belt. The shape of the dome was calculated in Rylov (1977, 1985).

3. Electrons move along the outflow channel which consists of:

(a) The internal acceleration zone (IAZ) of the outflow channel which is a thin $\left(l<10^{-4} R\right)$ layer near the stellar surface, where (massive or massless) electrons are accelerated up to a Lorentz factor of the order $\gamma_{1} \simeq 2 /\left(3 \sin ^{2} \nu_{b}\right)$, where $R \sin \nu_{b}$ is the radius of IAZ. As the calculation shows (Rylov 1979) $\gamma_{1}<$ $10^{3}$ to $10^{4}$.

(b) The inertial zone (IZ) occupies the main part of the outflow channel inside the dome. It is the capture region. Here besides the DP electrons with $\gamma=\gamma_{1}$, there are SP electrons, whose electric field compensates the longitudinal electric field. The DP electrons are not accelerated. The lectron motion inside the IZ was calculated in papers by Rylov $(1979,1987)$.

(c) In the external acceleration zone (EAZ) electrons are accelerated mainly towards the star. The Lorentz factor of (massive) electrons achieves $10^{8}$ to $10^{9}$ (Rylov 1977). The idea of returning electrons was presented by Jackson (1976). The motion of returning electrons was calculater by Rylov (1977).

The motion of electrons in the outflow channel can be stationary. But this steady-state motion is unstable. A very irregular motion arises as a result of interaction between $S P$ and $D P$ inside the outflow channel (Rylov 1987). This irregular flow is the basis of the pulsar radio emission. The last can be considered as a result of operating the vacuum-tube device "pulsar".

\section{Secondary effects}

The pulsar operation is followed by some secondary effects which can modify the pulsar parameters as a vacuum-tube device.

\section{Electron-positron pair production}

The flux of returning electrons emits hard $\gamma$-quanta which produce electron-positron pairs in the vicinity of the neutron star. Most of them fall onto the stellar surface, but some of them remain in the pulsar magnetosphere. As a result a conductive cloud of plasma arises around the star. The effective radius $R$ of the star increases. This change of the spatial scale leads to a change in the pulsar parameters. The increase of $R$ leads to a decrease of $\gamma$ quanta energy and to a decrease in pair production. The balanced effective radius $R$ depends on the pulsar parameters but does not exceed 10 stellar radii (Rylov 1981, Rylov 1984).

\section{Escaping hard charged particles from the pulsar magnetosphere}

Some of the positrons produced leave the electron dome. Then they are accelerated away from the star up to large Lorentz factors (of the order $10^{5}$ to $10^{9}$ ) and escape the pulsar magnetosphere. They do not return to the star as massless particles do, because at large distances $e \phi$ is less than the positron energy and the massless approximation is not valid for hard positrons. The positron escape leads to a decrease in the system charge $Q$, which leads to a compensating escape of electrons. So the electron-positron flow from the star is a result of pair production and of the violation of the massless approximation. 